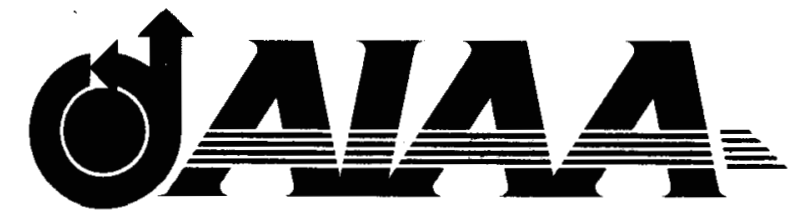

\title{
AIAA 99-3334
}

Distributed Relaxation Multigrid and Defect Correction Applied to the Compressible Navier-Stokes Equations

J. L. Thomas

NASA Langley Research Center Hampton, Virginia 23681

B. Diskin

ICASE NASA Langley Research Center Hampton, Virginia 23681 A. Brandt

The Weizmann Institute of Science Rehovot 76100, Israel

\section{4th Computational Fluid Dynamics Conference 28 June - 1 July, 1999/Norfolk, VA}




\title{
Distributed Relaxation Multigrid and Defect Correction Applied to the Compressible Navier-Stokes Equations
}

\author{
J. L. Thomas* \\ NASA Langley Research Center \\ Hampton, Virginia 23681 \\ B. Diskin \\ ICASE NASA Langley Research Center \\ Hampton, Virginia 23681 \\ A. Brandt \\ The Weizmann Institute of Science \\ Rehovot 76100, Israel \\ $6 / 28 / 99$
}

\begin{abstract}
The distributed-relaxation multigrid and defectcorrection methods are applied to the twodimensional compressible Navier-Stokes equations. The formulation is intended for high Reynolds number applications and several applications are made at a laminar Reynolds number of 10,000. A staggeredgrid arrangement of variables is used; the coupled pressure and internal energy equations are solved together with multigrid, requiring a block $2 \times 2$ matrix solution. Textbook multigrid efficiencies are attained for incompressible and slightly compressible simulations of the boundary layer on a flat plate. Textbook efficiencies are obtained for compressible simulations up to Mach numbers of 0.7 for a viscous wake simulation.
\end{abstract}

\section{Introduction}

Computational fluid dynamics (CFD) is becoming a more important part of the complete aircraft design cycle because of the availability of faster computers with more memory and improved numerical algorithms. The cruise shapes of transport aircraft are designed to minimize viscous and shock wave losses at transonic speeds and computational methods for these flows are reasonably well in hand. Simulations of off-design performance associated with maximum lift, buffet, and flutter and the deter-

*Head, Aerodynamic and Acoustic Methods Branch (AAMB), AIAA Fellow.

Copyright (C)1999 by the American Institute of Aeronautics and Astronautics, Inc. No copyright is asserted in the United States under Title 17, U.S. Code. The U.S. Government has a royalty-free license to exercise all rights under the copyright claimed herein for government purposes. All other rights are reserved by the copyright owner. mination of stability and control derivatives, involving unsteady separated and vortical flows with stronger shock waves, demand significant computing resources since Reynolds-averaged Navier-Stokes (RANS) methods are needed. The turnaround time of these computations for high-Reynolds flows over complex geometries is too long to impact the design cycle and the turbulence models for separated flows have a high degree of variability.

The current RANS solvers with multigrid require on the order of 1500 residual evaluations to converge the lift and drag to one percent of their final values for wing-body geometries near transonic cruise conditions. Complex geometry and complex physics simulations require many more residual evaluations to converge, if indeed convergence can even be attained. It is well-known for elliptic problems that solutions can be attained optimally using a full multigrid (FMG) process in far fewer, on the order of $2-4$, residual evaluations. An optimally convergent method is defined by Brandt ${ }^{1,2}$ as textbook multigrid efficiency (TME), meaning the solutions to the governing system of equations are attained in a computational work which is a small (less than 10) multiple of the operation count in the discretized system of equations. Thus, there is a potential gain of two orders of magnitude in operation count reduction if TME could be attained for the RANS equation sets. This possible two order of magnitude improvement in convergence represents an algorithmic floor since it is unlikely that faster convergence for these nonlinear equations could be attained. This algorithmic acceleration, however, coupled with further increases in computational speed can open up avenues and accelerate progress in many areas, including: the ap- 
plication of steady and time-dependent simulations in the high-lift, off-design, and stability and control areas; the usage of RANS solvers in the aerodynamic and multidisciplinary design areas; and the development of improved turbulence models.

The RANS equation sets are a system of coupled nonlinear equations which are not, even for subsonic Mach numbers, fully elliptic, but contain hyperbolic partitions. Brandt ${ }^{1}$ has summarized the progress and remaining barriers in TME for the equations of fluid dynamics. Although TME demonstrations, especially for incompressible simulations, have been completed, the distributed relaxation approach has not been used widely for large-scale simulations. Current methodologies use a block-matrix relaxation and/or a time-dependent approach to solve the equations; significant improvements have been demonstrated using multigrid approaches, but the methods are not optimally convergent. An example is the plane solver of Thomas, et al. ${ }^{3}$ which gives fast multigrid performance, but at a cost of block matrix solutions of size $m$, where $m$ is the number of conservation equations at a grid point. A subtle disadvantage of this block-matrix approach is that classical Gauss-Seidel relaxation methods, well suited for the constituent hyperbolic and elliptic partitions in the equations, cannot be applied stably in subsonic flow.

The distributed relaxation approach of Brandt decomposes the system of equations into separable, many times scalar, pieces that can be treated with optimal methods. The purpose of this paper is to apply distributed relaxation in a defect correction setting to the compressible Navier-Stokes equations. A staggered-grid discretization is used which simplifies the elements of the calculation, although this is not an essential ingredient. The method follows closely the original derivation of Brandt,,$^{1,2}$ dating to 1984 .

\section{Distributed Relaxation}

The equations for the time-dependent conservation of mass, momentum, and energy can be written as

$$
\partial_{t} \mathbf{Q}+\mathbf{R}=\mathbf{0}
$$

where $\mathbf{Q}=(\rho, \rho u, \rho v, e)^{T}$ and $\mathbf{R}(\mathbf{Q})$ is the spatial divergence of a vector function representing convection and viscous/heat transfer effects. The quantity $e$ is the total energy per unit volume, $\epsilon$ is the internal energy per unit mass, and the perfect gas equation of state relating pressure, $p$, to density, $\rho$, and $\epsilon$ can be written as

$$
p=(\gamma-1)\left[e-\rho\left(u^{2}+v^{2}\right) / 2\right]=(\gamma-1) \rho \epsilon,
$$

where $\gamma$ is the ratio of specific heats. A set of nonconservative equations can be cast in terms of primitive variables $q=(u, v, p, \epsilon)^{T}$ by multiplying the equations by the Jacobian matrix $\frac{\partial \mathbf{q}}{\partial \mathbf{Q}}$ given as

$$
\begin{gathered}
\frac{\partial \mathbf{q}}{\partial \mathbf{Q}}=\mathbf{S}\left[\begin{array}{cccc}
-1 & 1 & 0 & 0 \\
-1 & 0 & 1 & 0 \\
\left(u^{2}+v^{2}\right) / 2 & -u & -v & 1 \\
-\epsilon+\left(u^{2}+v^{2}\right) / 2 & -u & -v & 1
\end{array}\right] \\
\mathbf{S} \equiv\left[\begin{array}{cccc}
\rho & 0 & 0 & 0 \\
0 & \rho & 0 & 0 \\
0 & 0 & \gamma-1 & 0 \\
0 & 0 & 0 & \rho
\end{array}\right]
\end{gathered}
$$

The primitive equations are then

$$
\partial_{t} \mathbf{q}+\frac{\partial \mathbf{q}}{\partial \mathbf{Q}} \mathbf{R}=0
$$

These equations may be linearized about a given state variable and written in delta form, $\delta \mathbf{q} \equiv$ $\mathbf{q}^{n+1}-\mathbf{q}^{n}$, as

$$
\mathbf{L} \delta \mathbf{q}=-\frac{\partial \mathbf{q}}{\partial \mathbf{Q}} \mathbf{R},
$$

where in the matrix operator $\mathbf{L}$, only the principal terms at the viscous and inviscid scales are retained. Both scales are essential for high Reynolds number simulations: the inviscid scales over most of the flowfield and the viscous scales in the thin viscous layers near bodies and in their wakes. Since we are interested here in solutions to the steady-state equations, we drop the time derivative. Additionally, the thin-layer approximation, in which only the viscous terms associated with variations in the coordinate normal to the body are retained, can further simplify the terms in $\mathbf{L}$. This approximation is widelyused for high Reynolds number applications and is used for the numerical calculations below, although the development does not rely on this approximation. Also, the formulation allows a conservative discretization of $\mathbf{R}$ although, for the results below, a nonconservative form for $\frac{\partial \mathbf{q}}{\partial \mathbf{Q}} \mathbf{R}$ is used.

The distributed relaxation method replaces $\delta \mathbf{q}$ by $\mathbf{M} \delta \mathbf{w}$ so that the resulting matrix $\mathbf{L M}$ becomes a diagonal or lower triangular matrix, as 


$$
\mathbf{L} \mathbf{M} \delta \mathbf{w}=-\frac{\partial \mathbf{q}}{\partial \mathbf{Q}} \mathbf{R} \equiv-\mathbf{r}
$$

The diagonal elements of $\mathbf{L M}$ are composed ideally of the separable components of the determinant of the matrix $\mathbf{L}$ and represent the elliptic or hyperbolic features of the equation.

The approach yields fast convergence for both steady and unsteady simulations if the constituent scalar diagonal operators in LM are solved with fast methods, such as multigrid for the elliptic partitions and upwind methods for convection. Brandt ${ }^{1}$ has derived a set of matrices $\mathbf{M}$ for the equations of fluid dynamics which provide a convenient lower triangular form based on a discretization of the equation of state. A variation of that formulation, in which the equation of state is an algebraic equation, is used below. The Appendix gives the matrices for the incompressible equations.

\section{Defect Correction}

Since Eq. (7) is written in delta form, it is natural to consider defect correction for the update, namely a lower-order discretization of the left side of the equations in order to simplify the construction of the implicit equations. The most common approximation for the driver operator is a first-order discretization for the convective and pressure (inviscid) contributions. For this approach, we can write the implicit scheme as

$$
[\mathbf{L} \mathbf{M}]_{d} \delta \mathbf{w}=-\mathbf{r}_{t}
$$

where the subscripts $t$ and $d$ denote some desired "target" and "driver" schemes on the right and left sides, respectively, of the equation.

The convergence of defect correction is known to be fast for elliptic equations. For hyperbolic equations, the convergence may be slow, even if the implicit equations are solved exactly. This slowdown was pointed out by Brandt ${ }^{5}$ and discussed in some detail in Brandt and Yavneh, ${ }^{4}$ Thomas, et. al, ${ }^{3}$ and Diskin and Thomas. ${ }^{7}$ For the second-order upwindbiased discretization corresponding to $\kappa=0$, defined subsequently, the asymptotic convergence rate is approximately 0.5 per defect-correction iteration. For a first-order driver approximation, the initial convergence may be quite slow; the number of iterations to get into the asymptotic convergence regime might grow on fine grids and for certain frequencies at the rate $h^{-1 / 3}$. This worst case behavior is usually not that harmful for steady-state calculations with moderate frequency content of the information imposed at inflow.

With a second-order accurate behavior of the driver operator such as the fully-upwind secondorder operator $(\kappa=-1)$, the convergence is quite fast, within three iterations at most, although on very coarse meshes, in which neither the driver nor the target operators retain discretely an order property over the whole of the domain, some large growth of errors may occur. An efficient half-space Fourier mode analysis is given in Diskin and Thomas ${ }^{7}$ which predicts quite closely the actual solution behavior for scalar equations.

\section{Inviscid Compressible Equations}

The inviscid compressible equations take a relatively simple form with the choice of primitive variables considered here. Using a nonconservative discretization of the steady equations, the update equation is defined as

$$
\mathbf{L} \delta \mathbf{q}=-\mathbf{r}=-(\mathbf{f}+\mathbf{L} \mathbf{q}),
$$

where $\mathbf{f} \equiv \mathbf{0}$, the matrix $\mathbf{L}$ is

$$
\mathbf{L}=\left[\begin{array}{cccc}
Q & 0 & \frac{1}{\rho} \partial_{x} & 0 \\
0 & Q & \frac{1}{\rho} \partial_{y} & 0 \\
\rho c^{2} \partial_{x} & \rho c^{2} \partial_{y} & Q & 0 \\
\frac{c^{2}}{\gamma} \partial_{x} & \frac{c^{2}}{\gamma} \partial_{y} & 0 & Q
\end{array}\right]
$$

and $Q \equiv u \partial_{x}+v \partial_{y}$. The last equation is decoupled from the first three, representing the convection of entropy along a streamline. The determinant of the matrix of operators,

$$
Q^{2}\left[Q^{2}-c^{2} \Delta\right]
$$

corresponds to an elliptic partition, represented by the full potential equation, and two convective partitions, generally recognized as the convection of entropy and vorticity. Taking the distribution matrix $\mathbf{M}$ as the cofactors of the third row of $\mathbf{L}$ divided by their common factor,

$$
\mathbf{M}=\left[\begin{array}{cccc}
1 & 0 & -\frac{1}{\rho} \partial_{x} & 0 \\
0 & 1 & -\frac{1}{\rho} \partial_{y} & 0 \\
0 & 0 & Q & 0 \\
0 & 0 & 0 & 1
\end{array}\right]
$$

the equations to solve for the "ghost" $\delta$ w variables are 


$$
\mathbf{L} \mathbf{M} \delta \mathbf{w}=-\mathbf{r},
$$

or

$$
\begin{aligned}
Q \delta w_{1} & =-r_{1} \\
Q \delta w_{2} & =-r_{2} \\
\left\{-\frac{1}{c^{2}} Q^{2}+\Delta\right\} \delta w_{3}= & +\frac{1}{c^{2}} r_{3}+\rho\left(\partial_{x} \delta w_{1}+\partial_{y} \delta w_{2}\right) \\
\frac{1}{c^{2}} Q \delta w_{4}= & -\frac{1}{c^{2}} r_{4}-\frac{1}{\gamma}\left(\partial_{x} \delta w_{1}+\partial_{y} \delta w_{2}\right) \\
& +\frac{1}{\rho \gamma} \Delta \delta w_{3}
\end{aligned}
$$

The last equation could be replaced with

$$
Q \delta q_{4}=-r_{4}-\frac{c^{2}}{\gamma}\left(\partial_{x} \delta u+\partial_{y} \delta v\right)
$$

for inviscid flow. Thus the update to $\mathbf{q}$ is

$$
\begin{aligned}
u^{n+1}=u^{n}+\delta q_{1} & =u^{n}+\delta w_{1}-\frac{1}{\rho} \partial_{x} \delta w_{3} \\
v^{n+1}=v^{n}+\delta q_{2} & =v^{n}+\delta w_{2}-\frac{1}{\rho} \partial_{y} \delta w_{3} \\
p^{n+1}=p^{n}+\delta q_{3} & =p^{n}+Q \delta w_{3} \\
\epsilon^{n+1}=\epsilon^{n}+\delta q_{4} & =\epsilon^{n}+\delta w_{4}
\end{aligned}
$$

The staggered-grid discretization used here is usual: $p, \epsilon, \rho$ defined at the interiors of the grid, $u$ defined at the cell interfaces tangent to the $y$ - or $k$-direction, and $v$ defined at the cell interfaces tangent to the $x-$ or $j$-direction. The discrete scheme with such a staggered-grid arrangement of variables can be described as

$$
\mathbf{L}^{h}=\left[\begin{array}{cccc}
Q^{h} & 0 & \frac{1}{\rho} \partial_{x}^{h} & 0 \\
0 & Q^{h} & \frac{1}{\rho} \partial_{y}^{h} & 0 \\
\rho c^{2} \partial_{x}^{h} & \rho c^{2} \partial_{y}^{h} & \tilde{Q}_{0}^{h} & 0 \\
\frac{c^{2}}{\gamma} \partial_{x}^{h} & \frac{c^{2}}{\gamma} \partial_{y}^{h} & 0 & Q^{h}
\end{array}\right]
$$

where $\partial_{x}^{h}$ and $\partial_{x}^{h}$ are 2-point centered differences on the staggered grid. The operator $Q^{h}$ is an upwindbiased convection operator, defined in terms of translation operators $T_{j}$ and $T_{k},\left(T_{j} u_{j, k}=u_{j+1, k}\right)$, as

$$
\begin{aligned}
Q^{h}= & \frac{u^{+}}{\left(h_{x}\right)_{j}} D^{-}\left(T_{j}\right)+\frac{u^{-}}{\left(h_{x}\right)_{j}} D^{+}\left(T_{j}\right) \\
& +\frac{v^{+}}{\left(h_{y}\right)_{k}} D^{-}\left(T_{k}\right)+\frac{v^{-}}{\left(h_{y}\right)_{k}} D^{+}\left(T_{k}\right) \\
D^{-}\left(T_{j}\right) \equiv & c_{1} T_{j}^{-2}+c_{2} T_{j}^{-1}+c_{3}+c_{4} T_{j}^{+1} \\
D^{+}\left(T_{j}\right) \equiv & -c_{1} T_{j}^{+2}-c_{2} T_{j}^{+1}-c_{3}-c_{4} T_{j}^{-1} \\
u^{ \pm} \equiv & (u \pm|u|) / 2
\end{aligned}
$$

The coefficients for $\kappa \in[-1,1]$ are

$$
\left\{c_{1}, c_{2}, c_{3}, c_{4}\right\}=\frac{1}{4}\{1-\kappa, 3 \kappa-5,3(1-\kappa), 1+\kappa\}
$$

and for first-order accuracy are

$$
\left\{c_{1}, c_{2}, c_{3}, c_{4}\right\}=\{0,-1,1,0\} .
$$

The convection operator in the pressure equation is differenced with a downwind operator, i.e.,

$$
\begin{aligned}
\tilde{Q}_{0}^{h}= & \frac{u^{+}}{\left(h_{x}\right)_{j}} D^{+}\left(T_{j}\right)+\frac{u^{-}}{\left(h_{x}\right)_{j}} D^{-}\left(T_{j}\right) \\
& +\frac{v^{+}}{\left(h_{y}\right)_{k}} D^{+}\left(T_{k}\right)+\frac{v^{-}}{\left(h_{y}\right)_{k}} D^{-}\left(T_{k}\right)
\end{aligned}
$$

so that the determinant of the discrete matrix $\mathbf{L}^{h}$,

$$
\left(Q^{h}\right)^{2}\left[Q^{h} \tilde{Q}_{0}^{h}-c^{2} \Delta^{h}\right],
$$

has a smaller and more symmetrical stencil than if the upwind operator were used everywhere. For grid-aligned flow, this full potential operator is a centered 5-point discretization of the Prandtl-Glauert equation. With this formulation, the low-Mach number limit is not a problem, as the usual five-point Laplacian is obtained for the $w_{3}$ variable, analogous to the purely incompressible form.

\section{Compressible Navier-Stokes Equations}

The contributions to the principal terms of the matrix of operators for the viscous and heat conduction terms arise from the momentum and energy contributions as in Eq. (3), since there is no influence of viscosity in the continuity equation. The two major complications are that the heat conduction term couples into both the pressure and internal energy equations and the viscous terms in the momentum equations involve cross-derivative terms. With the nonconservative discretization considered here assuming constant viscosity and heat conduction coefficients, the update equation can be written 
as Eq. (9) with $\mathbf{f} \equiv-(0,0,(\gamma-1) \Phi, \rho \Phi)^{T}$ where the viscous dissipation term $\Phi$ is

$$
\Phi \equiv \mu\left[2\left(\partial_{x} u\right)^{2}+2\left(\partial_{y} v\right)^{2}+\left(\partial_{x} v+\partial_{y} u\right)^{2}\right]+\lambda\left[\left(\partial_{x} u+\partial_{y} v\right)^{2}\right]
$$

the matrix $\mathbf{L}$ is

$$
\mathbf{L}=\left[\begin{array}{cccc}
Q_{\nu}-\frac{\hat{\lambda}}{\rho} \partial_{x x} & -\frac{\hat{\lambda}}{\rho} \partial_{x y} & \frac{1}{\rho} \partial_{x} & 0 \\
-\frac{\hat{\lambda}}{\rho} \partial_{x y} & Q_{\nu}-\frac{\hat{\lambda}}{\rho} \partial_{y y} & \frac{1}{\rho} \partial_{y} & 0 \\
\rho c^{2} \partial_{x} & \rho c^{2} \partial_{y} & Q & -(\gamma-1) k \Delta \\
\frac{c^{2}}{\gamma} \partial_{x} & \frac{c^{2}}{\gamma} \partial_{y} & 0 & Q_{k / \rho}
\end{array}\right]
$$

and $Q_{\chi} \equiv Q-\chi \Delta$. Nondimensionalizing by density and speed of sound and using the Stokes hypothesis for the bulk viscosity term, the coefficients become $\nu=\mu / \rho=M_{\infty} /(\operatorname{Re} \rho), k=M_{\infty} \gamma /(\operatorname{RePr})$ and $\hat{\lambda}=$ $\lambda+\mu=\mu / 3$.

The distribution matrix $\mathbf{M}$ is developed similarly to that for the inviscid equations with some additions that eliminate the cross-derivative terms, as below

$$
\mathbf{M}=\left[\begin{array}{cccc}
1 & 0 & -\frac{1}{\rho} \partial_{x} & 0 \\
0 & 1 & -\frac{1}{\rho} \partial_{y} & 0 \\
\hat{\lambda} \partial_{x} & \hat{\lambda} \partial_{y} & Q_{\nu+\frac{\lambda}{\rho}} & 0 \\
0 & 0 & 0 & 1
\end{array}\right]
$$

The matrix LM now has only diagonal entries of $Q_{\nu}$ in the first two rows. The last two equations are still coupled, requiring a block $2 \times 2$ solution as below,

$$
\begin{gathered}
{\left[\begin{array}{cc}
-\frac{1}{c^{2}} Q Q_{\nu+\frac{\hat{\lambda}}{\rho}}+\Delta & \frac{(\gamma-1) k}{c^{2}} \Delta \\
-\frac{1}{\rho \gamma} \Delta & Q_{k / \rho}
\end{array}\right]\left[\begin{array}{l}
\delta w_{3} \\
\delta w_{4}
\end{array}\right]} \\
=\left[\begin{array}{l}
g_{3} \\
g_{4}
\end{array}\right]
\end{gathered}
$$

where

$$
\begin{aligned}
g_{3}= & \frac{1}{c^{2}} r_{3}+\rho\left(\partial_{x} \delta w_{1}+\partial_{y} \delta w_{2}\right) \\
& +\frac{\hat{\lambda}}{c^{2}} Q\left(\partial_{x} \delta w_{1}+\partial_{y} \delta w_{2}\right) \\
g_{4}= & -\frac{1}{c^{2}} r_{4}-\frac{1}{\gamma}\left(\partial_{x} \delta w_{1}+\partial_{y} \delta w_{2}\right)
\end{aligned}
$$

In the high Reynolds number simulation considered here, the term $\left(-\frac{1}{c^{2}} Q Q_{\nu+\frac{\bar{\lambda}}{\rho}}+\Delta\right)$ in Eq. (16) is approximated as the full potential operator associated with the inviscid equations. The discrete approximation of the terms above are straightforward additions to the inviscid compressible terms, since the viscous terms are differenced centrally.

In the calculations below, the thin-layer approximation is made for the viscous terms, i.e., only the derivative terms in the $y$-direction are retained. The $\delta w_{1}$ and $\delta w_{2}$ equations (the momentum equations at constant pressure) are solved with a tridiagonal $y$ line marching algorithm. The equations for $\delta w_{3}$ and , $\delta w_{4}$ are solved with two passes of a $\mathrm{V}(2,1)$ multigrid cycle with line Gauss-Seidel iteration using four multigrid levels; a correction scheme (CS) multigrid was used in which the coefficients of the block matrix terms were interpolated to cell center locations using bilinear interpolation. The coarsest grid level was solved exactly. A smoothing was done with a point Gauss-Seidel scheme before transferring residuals to coarser meshes.

\section{Computational Results}

\section{Inviscid Linearized Flow}

The linearized flow over a bump in a channel was computed for a computational domain extending from $x=0$ to $x=3$ and $y=0$ to $y=1$ on a uniform grid of mesh size $h_{x}=h_{y}$. A sine-squared profile extending from $x=1$ to $x=2$ of height 0.1 that of the channel height was imposed with linearized boundary conditions, $v / u_{\infty}=d y / d x$ at $y=0$.

The boundary conditions for the primitive and ghost variables require some discussion. The residuals at the horizontally oriented boundaries are taken to be zero, corresponding to an imposed boundary condition of tangency. In general, satisfaction of these residuals to zero is obtained by adjusting the pressure across the boundary to satisfy the normalmomentum equation. The boundary condition imposed on the $\delta w_{3}$ ghost variable along the top and bottom of the domain is $\partial_{y} \delta w_{3}=0$, corresponding to the prescribed normal velocity condition, $\delta v=0$.

The residuals and $u$ at the vertically oriented upstream boundary are unknowns in the solution; the variables $p, \epsilon$ and $u$ at points just upstream of the boundary are taken as the imposed boundary conditions; the upstream boundary conditions imposed in the ghost-variable solutions are as follows:

$$
\delta w_{1}=0 ; \delta w_{3}=0 ; \delta w_{4}=0 .
$$

corresponding to imposed values of $u, p, e$. The value of $v$ upstream of the boundary can be determined by imposing $\delta w_{2}=0$ and then correcting it at values outside the domain using the update formulas. 


\begin{tabular}{|c|c|c|c|}
\hline$h_{x}=h_{y}$ & $C_{p}$, & $C_{p}$, & $C_{p}$, \\
\hline $1 / 4$ & -0.0058 & -0.0058 & -0.0074 \\
\hline $1 / 8$ & -0.1767 & -0.1767 & -0.2116 \\
\hline $1 / 16$ & -0.3164 & -0.3165 & -0.3730 \\
\hline $1 / 32$ & -0.3650 & -0.3651 & -0.4274 \\
\hline $1 / 64$ & -0.3785 & -0.3786 & -0.4423 \\
\hline
\end{tabular}

Table 1. Linearized pressure coefficients at $x=1.5$ for the flow over a sine-squared bump in a channel.

The pressures at locations just downstream of the downstream boundary are specified as freestream; all of the other values are obtained through integration of the update equations with the upwind-biased convection operator. The downstream boundary conditions imposed on the ghost variable $\delta w_{3}$ is $\partial_{x} \delta w_{3}=0$, corresponding to the prescribed pressure condition, $\delta p=0$. Thus, in summary, Dirichlet conditions are imposed for $\delta \mathbf{w}$ at the upstream boundary and Neumann conditions for $\delta w_{3}$ at all other boundaries.

The exact discrete solution for both incompressible and compressible flow is obtained in one iteration starting from freestream conditions if (1) we solve the $\delta w_{3}$ equation to machine zero using conventional multigrid methods and (2) we use firstorder differencing. We are not recommending that residuals be reduced to machine zero; rather this approach does provide a convenient check of the implementation of the algorithm on the computer, since arbitrarily large perturbations in $\mathbf{q}$ can be damped/convected from the domain in one iteration. The ghost variable $\delta w_{3}$ in the domain takes the role of the perturbation potential in an irrotational isentropic flow. The values of $u$ and $v$ are obtained to second order. The value of $p$ is determined by the accuracy of the convection operator. Fast convergence and second-order accuracy is obtained if we use defect correction with the second-order convection operator $(\kappa=0)$. The values of $C_{p} \approx-2\left(u-u_{\infty}\right)$ at $x=1.5$ obtained on a sequence of grids are shown in Table 1. Second-order accuracy is obtained and the results obey the expected Prandtl-Glauert scaling of the pressure coefficient with $\left.1 / \sqrt{(1}-M_{\infty}^{2}\right)$; the $C_{p}$ decrease for $M_{\infty}=0.5$ is 1.17 , as compared to 1.16 from the Prandtl-Glauert scaling.

\section{Viscous Flat Plate}

The viscous flow over a flat plate was computed for the same computational domain as above at a $\operatorname{Re}=10,000$ based on the height of the channel. The grid was stretched in the $y$-direction with a stretching factor of 1.06 on the finest mesh of $N_{x} \times N_{y}=$ 193x65. No-slip, adiabatic wall conditions are prescribed from $x=1$ to $x=2$ along the lower bound ary and symmetry conditions upstream and downstream of those points; a wake profile develops downstream of the trailing edge $x=2$. Pressure was prescribed at the downstream boundary and tangency along the upper wall.

The ghost-variable boundary conditions are the same as the channel flow simulation except in the region of the flat plate. For the internal energy, the gradient normal to the boundary is zero for both the adiabatic and symmetry conditions, so that $\partial_{y} \delta w_{4}=0$ is imposed. Since the residuals and $v$ are prescribed to be zero at the lower boundary, the boundary conditions for the $\delta w_{3}$ variable is taken as $\partial_{y} \delta w_{3}=0$. Thus, boundary conditions for the ghost variables in this simulation are the same as the channel flow simulation. The primitive variable velocities outside the lower boundary in the region of the plate are chosen to satisfy the no-slip condition at the plate, i.e. $u(-y)=-u(y) ; v(-y)=-v(y)$.

Convergence could be attained in this simulation for incompressible and slightly compressible flow. An FMG cycle was used. The value of $C_{f}$ at $x=1.5$, midway down the plate, is a sensitive measure of convergence. First-order solutions were obtained which decreased the $L_{2}$-norm of the maximum residual 4-5 orders of magnitude on each of the three finer meshes over 20 iterations, corresponding to a convergence rate of roughly 0.6 per fine-grid iteration for both incompressible flow and $M_{\infty}=0.1$. The skin friction values converged to within discretization error in 2-4 cycles. For second-order accuracy of the convection operator, $\kappa=0$, skin friction results are tabulated in Table 2 for the series of meshes and for 2,4,10, and 20 fine-grid iterations. The convergence is quite fast; solutions are obtained to within truncation-error accuracy within a few multigrid cycles. The $L_{2}$-norm of the maximum residual was reduced 3 orders of magnitude on each of the three finer meshes in 20 iterations, corresponding to a convergence rate of roughly 0.7 per fine-grid iteration, again for both incompressible flow and $M_{\infty}=0.1$.

At higher Mach numbers, convergence could not be attained. The residuals remained quite large and the solution eventually diverged near the leading edge of the plate. This behavior is not entirely unexpected since we expect the distributed relaxation approach to be augmented with procedures to locally reduce the residuals near boundaries, before relaxing the interior equations. This local relaxation near boundaries is required to guarantee convergence within a few cycles even for elliptic equations. ${ }^{6}$ 


\begin{tabular}{|c|c|c|c|}
\hline$N_{x} \times N_{y}$ & Iterations & $\begin{array}{c}C_{f}, \\
M_{\infty}=0\end{array}$ & $\begin{array}{c}C_{f}, \\
M_{\infty}=0.1\end{array}$ \\
\hline $25 \times 9$ & 2 & $0.973 \mathrm{E}-02$ & $0.111 \mathrm{E}-01$ \\
\hline $25 \times 9$ & 4 & $0.392 \mathrm{E}-02$ & $0.685 \mathrm{E}-02$ \\
\hline $25 \times 9$ & 10 & $0.490 \mathrm{E}-02$ & $0.574 \mathrm{E}-02$ \\
\hline $25 \times 9$ & 20 & $0.482 \mathrm{E}-02$ & $0.566 \mathrm{E}-02$ \\
\hline \hline $49 \times 17$ & 2 & $0.694 \mathrm{E}-02$ & $0.756 \mathrm{E}-02$ \\
\hline $49 \times 17$ & 4 & $0.780 \mathrm{E}-02$ & $0.831 \mathrm{E}-02$ \\
\hline $49 \times 17$ & 10 & $0.813 \mathrm{E}-02$ & $0.875 \mathrm{E}-02$ \\
\hline $49 \times 17$ & 20 & $0.814 \mathrm{E}-02$ & $0.877 \mathrm{E}-02$ \\
\hline \hline $97 \times 33$ & 2 & $0.924 \mathrm{E}-02$ & $0.934 \mathrm{E}-02$ \\
\hline $97 \times 33$ & 4 & $0.937 \mathrm{E}-02$ & $0.949 \mathrm{E}-02$ \\
\hline $97 \times 33$ & 10 & $0.931 \mathrm{E}-02$ & $0.948 \mathrm{E}-02$ \\
\hline $97 \times 33$ & 20 & $0.931 \mathrm{E}-02$ & $0.948 \mathrm{E}-02$ \\
\hline \hline $193 \times 65$ & 2 & $0.970 \mathrm{E}-02$ & $0.970 \mathrm{E}-02$ \\
\hline $193 \times 65$ & 4 & $0.967 \mathrm{E}-02$ & $0.971 \mathrm{E}-02$ \\
\hline $193 \times 65$ & 10 & $0.960 \mathrm{E}-02$ & $0.967 \mathrm{E}-02$ \\
\hline $193 \times 65$ & 20 & $0.959 \mathrm{E}-02$ & $0.967 \mathrm{E}-02$ \\
\hline
\end{tabular}

Table 2. Convergence of the skin friction value halfway down the viscous flat plate for incompressible and slightly compressible flow; $R e=10,000$.

\section{Viscous Wake}

In order to investigate the behavior of the viscous algorithm for higher Mach numbers in a smoother flow, the viscous flow in a developing wake profile was considered. The simulation was similar to that above except that a wake deficit with uniform pressure and internal energy was applied at the inflow boundary. The inlet profile at $x=-h_{x}$ was prescribed according to the exact incompressible far wake solution, as

$$
u / u_{\infty}=1-w_{d}(x+1)^{-1 / 2} \exp \left(\frac{-\operatorname{Re} y^{2}}{4(x+1)^{2}}\right)
$$

where $w_{d}=0.5$. The minimum velocity was monitored at $x=1.5$, a location midway in the domain, as a measure of convergence. The boundary conditions for the ghost variables are the same as the channel flow simulation.

Convergence could be attained from incompressible flow up to the highest Mach number investigated, $M_{\infty}=0.7$. First-order solutions were obtained which decreased the $L_{2}$-norm of the maximum residual 5 orders of magnitude on each of the three finer meshes over 10 iterations, corresponding to a convergence rate of roughly 0.3 per fine-grid iteration at all Mach numbers The wake deficit values converged to within discretization error in 2 iterations. For second-order accuracy of the convection

\begin{tabular}{|c|c|c|c|}
\hline$N_{x} \times N_{y}$ & Iterations & $\begin{array}{c}u_{\min }, \\
M_{\infty}=0\end{array}$ & $\begin{array}{c}u_{\min }, \\
M_{\infty}=0.7\end{array}$ \\
\hline $25 \times 9$ & 1 & 0.7149 & 0.7149 \\
\hline $25 \times 9$ & 2 & 0.7116 & 0.7141 \\
\hline $25 \times 9$ & 4 & 0.7105 & 0.7112 \\
\hline $25 \times 9$ & 10 & 0.7105 & 0.7112 \\
\hline \hline $49 \times 17$ & 1 & 0.7474 & 0.7478 \\
\hline $49 \times 17$ & 2 & 0.7483 & 0.7487 \\
\hline $49 \times 17$ & 4 & 0.7482 & 0.7485 \\
\hline $49 \times 17$ & 10 & 0.7481 & 0.7485 \\
\hline \hline $97 \times 33$ & 1 & 0.7520 & 0.7523 \\
\hline $97 \times 33$ & 2 & 0.7531 & 0.7534 \\
\hline $97 \times 33$ & 4 & 0.7530 & 0.7533 \\
\hline $97 \times 33$ & 10 & 0.7530 & 0.7533 \\
\hline \hline $193 \times 65$ & 1 & 0.7529 & 0.7532 \\
\hline $193 \times 65$ & 2 & 0.7532 & 0.7535 \\
\hline $193 \times 65$ & 4 & 0.7531 & 0.7535 \\
\hline $193 \times 65$ & 10 & 0.7531 & 0.7534 \\
\hline
\end{tabular}

Table 3. Convergence of the minimum velocity at $x=1.5$ for incompressible and compressible wake flows; $R e=10,000$.

operator, $\kappa=0$, minimum velocity values are tabulated in Table 3 for the series of meshes used in the FMG cycle and for 1,2,4, and 10 fine-grid iterations. The solution converges to within truncationerror accuracy within 2 multigrid cycles. The $L_{2}$ norm of the maximum residual was reduced 3 orders of magnitude on each of the three finer meshes in 10 iterations, corresponding to a convergence rate of roughly 0.5 per fine-grid iteration, again for both incompressible flow and $M_{\infty}=0.7$.

\section{Concluding Remarks}

The distributed-relaxation multigrid and defectcorrection methods are applied to the compressible Navier-Stokes equations. The formulation is intended for high Reynolds number applications and several applications are made at a laminar Reynolds number of 10,000 . Although deemed not essential to the methodology, both a staggered-grid arrangement of variables and nonconservative forms for the governing equations have been used.

The compressible flow algorithm solves a multigrid problem for the pressure and internal energy equations which necessitates a local block $2 \times 2$ matrix solution at every grid point. Since we obtain solutions to within truncation error in 2-4 fine-grid iterations and the additional operations to solve for the ghost variables are on the order of an additional residual evaluation, textbook multigrid efficiencies 
are attained for incompressible and slightly compressible simulations of the boundary layer on a flat plate. Difficulties were encountered for higher Mach numbers; the calculation can and should be augmented with local procedures to reduce the residuals near boundaries, since this approach is required for general conditions even for the elliptic equations. Textbook efficiencies are obtained for compressible simulations up to Mach numbers of 0.7 for a viscous wake simulation.

\section{Acknowledgment}

The first author wishes to thank T. Roberts of NASA and D. Sidilkover of ICASE for many interesting discussions of the distributed relaxation approach.

\section{References}

1. Brandt, A., "Guide to Multigrid Development," Multigrid Methods, Lecture Notes in Mathematics, Vol. 960, Springer-Verlag, 1984, pp. 220-317.

2. Brandt, A., "Barriers to Achieving Textbook Multigrid Efficiency (TME) in CFD," ICASE Interim Report No. 32, NASA/CR-1998-207647, 1998.

3. Thomas, J. L., Bonhaus, D. L., Anderson, W. K., Rumsey, C. L., and Biedron, R. T., "An $O\left(N m^{2}\right)$ Plane Solver for the Compressible Navier-Stokes Equations," AIAA 99-0785, 1999.

4. Brandt, A. and Yavneh, I., "On Multigrid Solution of High-Reynolds Incompressible Entering Flows," Journal of Computational Physics, Vol. 101, 1992, pp. 151-164.

5. Brandt, A., "Multigrid Solvers for Non-Elliptic and Singular Perturbation Problems," unpublished, The Weizmann Institute of Science, Rehovot, Israel, December 1981.

6. Brandt, A., "Rigorous Quantitative Analysis of Multigrid I: Constant Coefficients Two-Level Cycle with $L_{2}$-Norm," SIAM J. Numer. Anal., Vol. 31, No. 6, 1994, pp. 1695-1730.

7. Diskin, B., and Thomas, J. L., "Solving UpwindBiased Discretizations: Defect Correction Iterations," ICASE Report No. 99-12, NASA/CR1999-209106, 1999.

\section{Appendix - Incompressible Equations}

The update equations for the nonconservative incompressible viscous equations can be written as Eq. (6) with

$$
\mathbf{L} \equiv\left[\begin{array}{cccc}
Q_{\nu} & 0 & \partial_{x} & 0 \\
0 & Q_{\nu} & \partial_{y} & 0 \\
\partial_{x} & \partial_{y} & 0 & 0 \\
0 & 0 & 0 & Q_{k}
\end{array}\right]
$$

$\mathbf{f} \equiv-(0,0,0, \Phi)^{T}$, and $\lambda=0$. Nondimensionalized by density and velocity, the viscosity and heat conduction terms are $\nu=\mu / \rho=1 / R e$ and $k=\gamma /(\operatorname{Re} P r)$. The distribution matrix $\mathbf{M}$ is determined by the cofactors of the third row of $\mathbf{L}$ divided by their common factor, as

$$
\mathbf{M}=\left[\begin{array}{cccc}
1 & 0 & -\partial_{x} & 0 \\
0 & 1 & -\partial_{y} & 0 \\
0 & 0 & Q_{\nu} & 0 \\
0 & 0 & 0 & 1
\end{array}\right]
$$

The equations to solve for the ghost variables are $\mathbf{L} \mathbf{M} \delta \mathbf{w}=-\mathbf{r}$, as

$$
\begin{aligned}
Q_{\nu} \delta w_{1} & =-r_{1} \\
Q_{\nu} \delta w_{2} & =-r_{2} \\
\Delta \delta w_{3} & =+r_{3}+\partial_{x} \delta w_{1}+\partial_{y} \delta w_{2} \\
Q_{k} \delta w_{4} & =-r_{4}
\end{aligned}
$$

The distribution matrix $\mathbf{M}$ has the same form for inviscid flow $(\nu=0)$ as viscous flow. The distribution formulas reflect the role of pressure in the incompressible equations to satisfy the continuity equation. 